\title{
Behavioral Properties of the Trigeminal Somatosensory System in Rats Performing Whisker-Dependent Tactile Discriminations
}

\author{
David J. Krupa, Matthew S. Matell, Amy J. Brisben, Laura M. Oliveira, and Miguel A. L. Nicolelis \\ Department of Neurobiology, Duke University Medical Center, Durham, North Carolina 27710
}

To address several fundamental questions regarding how multiwhisker tactile stimuli are integrated and processed by the trigeminal somatosensory system, a novel behavioral task was developed that required rats to discriminate the width of either a wide or narrow aperture using only their large mystacial vibrissae. Rats quickly acquired this task and could accurately discriminate between apertures of very similar width. Accurate discriminations required a large number of intact facial whiskers. Systematic removal of individual whiskers caused a decrease in performance that was directly proportional to the number of whiskers removed, indicating that tactile information from multiple whiskers is integrated as rats gauge aperture width. In different groups of rats, different sets of whiskers were removed in patterns that preferentially left whisker rows or whisker arcs intact. These different whisker removals caused similar decreases in performance, indicating that individual whiskers within the vibrissal array are functionally equivalent during performance of this task. Lesions of the barrel cortex abolished the ability of rats to discriminate, demonstrating that this region is critically involved in this tactile behavior. Interestingly, sectioning the facial nerve, which abolished whisker movements, did not affect the ability to perform accurate discriminations, indicating that active whisker movements are not necessary for accurate performance of the task. Collectively, these results indicate that the trigeminal somatosensory system forms internal representations of external stimuli (in this case, aperture width) by integrating tactile input from many functionally equivalent facial whiskers and that the vibrissal array can function as a fine-grained distance detector without active whisker movements.

Key words: vibrissa; whisker; somatosensation; whisking; sensory integration; rat
The large mystacial vibrissae of the rat are arranged in a characteristic, highly conserved array of five rows and up to seven arcs (Woolsey and Van der Loos, 1970; Welker, 1971; Brecht et al., 1997). Rats can use these facial whiskers to perform a variety of tactile discriminations and behaviors (Vincent, 1912; Gustafson and Felbain-Keramidas, 1977; Hutson and Masterton, 1986; Carvell and Simons, 1990). However, several very fundamental questions regarding important aspects of whisker-dependent behaviors remain largely unanswered.

For instance, there is some controversy regarding whether the vibrissal array functions as a skin-like receptive surface used for fine-grained texture discriminations (Carvell and Simons, 1990) or, alternatively, as a specialized distance-detector sense organ (Brecht et al., 1997). Although the vibrissal array may perform both functions, no study has systematically demonstrated that rats are capable of performing fine-grained distance discriminations using only their mystacial vibrissae. Here, we tested and quantified the ability of rats to perform very fine-grained distance discriminations.

A second question addressed here was whether integration of tactile input from many whiskers is necessary for rats to perform

\footnotetext{
Received March 14, 2001; revised May 10, 2001; accepted May 11, 2001.

This work was supported by National Institutes of Health Grant DE-11121 to M.A.L.N. and an individual National Research Service Award MH-11808 to D.J.K. We thank Drs. Mark Laubach and Rodney Swain for helpful suggestions during development of the behavioral task, Ning Ling for assistance in training rats, and Dr. Christine Logan and Marshall Shuler for assistance and comments during preparation of this manuscript.

Correspondence should be addressed to Dr. David J. Krupa, Department of Neurobiology, Box 3209, Room 333, Duke University Medical Center, Bryan Research Building, 101 Research Drive, Durham, NC 27710. E-mail: krupa@neuro.duke.edu.

Copyright (C) 2001 Society for Neuroscience 0270-6474/01/215752-12\$15.00/0
}

the discrimination. Neurons in the trigeminal somatosensory system can respond to stimulation of a large number of individual whiskers (Ito, 1985; Chapin, 1986; Armstrong-James and Fox, 1987; Chiaia et al., 1991; Nicolelis and Chapin, 1994; Moore and Nelson, 1998; Ghazanfar and Nicolelis, 1999; Zhu and Connors, 1999). Thus, at the single-neuron level, there is a high degree of integration of tactual input from multiple whiskers. In contrast, evidence that rats integrate tactile input from many whiskers to perform whisker-dependent behaviors is lacking. Thus, it remains possible that, at the behavioral level, tactual input from individual whiskers is processed and perceived relatively independently with little or no multiwhisker integration, a possibility consistent with the one-to-one anatomical mapping of the vibrissal array throughout multiple levels of the trigeminal system (Woolsey and Van der Loos, 1970; Welker, 1971; Killackey, 1973; Belford and Killackey, 1979; Arvidsson, 1982; Land and Simons, 1985).

We also examined the functional similarity between different whiskers. Individual whiskers may be relatively equivalent in function, similar to localized patches of skin on a fingertip (Carvell and Simons, 1990). In contrast, it is possible that different whiskers form functionally distinct components of larger functional units such as whisker rows or whisker arcs (Brecht et al., 1997). No previous behavioral study has systematically examined the degree to which different facial whiskers or whisker rows and arcs are functionally similar or distinct.

We also investigated the role of active whisker movement in tactile discriminations. Rats use "whisking" motions of their mystacial vibrissae to explore novel objects (Welker, 1964) or to perform certain tactile discriminations (Carvell and Simons, 1990). Hypotheses regarding neuronal processing in the trigeminal system suggest a critical role for active whisking (Simons, 
1995; Moore et al., 1999). However, it remains unclear whether whisking is necessary for fine-grained tactile discriminations.

Finally, a previous report indicated that barrel cortex is critically involved in detecting certain whisker stimuli but not others (Hutson and Masterton, 1986). Thus, we examined whether barrel cortex was critically involved in accurate performance of these tactile discriminations.

\section{MATERIALS AND METHODS}

To test the issues described above, rats were trained to perform a novel behavioral task that required them to discriminate between a "wide" or "narrow" aperture using only their large mystacial vibrissae (see Figs. 1, 2; see below). Here, the large mystacial vibrissae are defined as the facial whiskers in rows A-E and arcs 1-7 and the four straddler whiskers (for instance, see Fig. 1 of Arvidsson, 1982). In different groups of rats, different manipulations of either the vibrissal array or the trigeminal somatosensory system were used to examine quantitatively a number of issues related to the behavioral properties associated with processing tactile input by the trigeminal system (described in detail below).

The apparatus in which this task was performed was designed to eliminate the possibility that rats might use other sensory cues (for instance, visual or olfactory) to solve the task or, more important, that the rats might use the smaller whiskers around their lip and nose instead

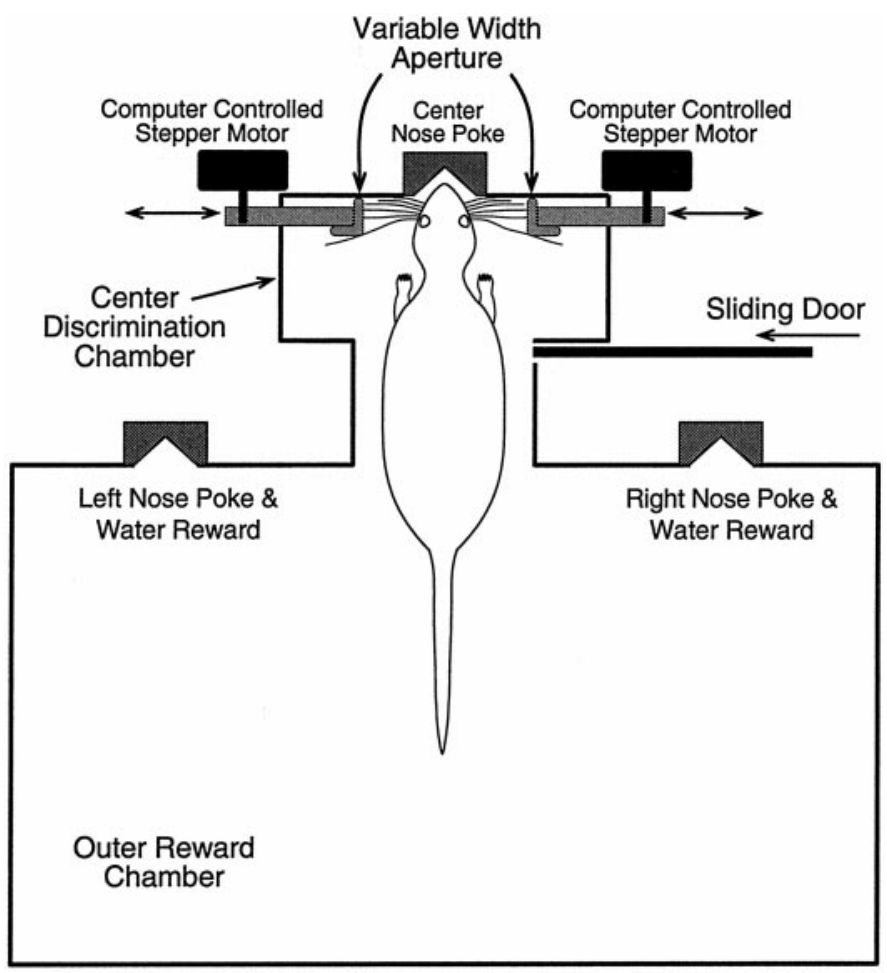

Figure 1. Schematic diagram of the behavioral training apparatus. The width of the variable-width aperture is set by rotating the computercontrolled stepper motors. Moving each aperture inward, toward the center, results in a narrower aperture; moving them outward results in a wider aperture. At the start of each training session, rats are placed in the outer reward chamber with the sliding door closed. When the door is opened, rats enter the center discrimination chamber and poke their nose into the center nose poke and sample the variable-width aperture with their facial whiskers. After poking their nose into the center nose poke (detected by interrupting an infrared photobeam), rats then back out into the outer reward chamber and poke their nose into either the left or right reward nose poke to receive a water reward, a left nose poke if the aperture is narrow and a right nose poke if the aperture is wide. Immediately after rats poke their nose into either the left or right nose poke, the sliding door between the outer reward chamber and the center discrimination chamber is closed. A new trial begins when the sliding door is again opened. of their large facial whiskers to perform the tactile discriminations. Furthermore, the behavioral apparatus was designed so that rats would approach and sample the variable-width aperture with their large facial whiskers in a well controlled and very repeatable manner.

Behavioral training apparatus. The behavioral apparatus (Fig. 1) consists of a large outer reward chamber attached to a smaller center discrimination chamber by a narrow passage. The center discrimination chamber is separated from the outer reward chamber by a sliding door. Along the front wall of the outer reward chamber are two nose pokes (Fig. 1) that allow the rat to signal whether the aperture is wide or narrow (described in detail below). Within each of these nose pokes is a $2 \mathrm{~mm}$ diameter tube that delivers drops of water as a reward for correct discriminations. Directly in front of each nose poke is a small door that completely covers the nose poke when closed, thereby preventing access to the poke (not shown in Fig. 1). Each nose poke has an infrared photobeam across the opening that detects whenever a rat pokes its nose into the nose poke. Breaking the photobeam sends a signal to a computer controlling the behavioral experiment (below). The center discrimination chamber contains a third nose poke located in the middle of the front wall (see Figs. 1,2) that also has an infrared photobeam across the opening that detects when a rat's nose has entered the center poke. The entire behavioral apparatus is located inside a sound-attenuating and light-proof isolation box.

Located in front of the center nose poke is the variable-width aperture (see Figs. 1, 2). The aperture consists of two pieces of right-angle shaped aluminum extrusions, $2.5 \mathrm{~cm}$ wide and $10 \mathrm{~cm}$ long. These extrusions are mounted vertically to aluminum bars that are connected to computercontrolled stepper motors via a spur gear-rack and pinion assembly (Fig. 1). The aluminum bars ride on frictionless, linear sliders. When mounted this way, these extrusions create a narrow wall on either side of the center nose poke. By rotating the stepper motors, these aluminum bar-extrusion assemblies can be precisely moved inward or outward. Thus, by moving these extrusions away from the center, the aperture is made wider. Conversely, by moving the extrusions inward toward the center, the aperture is made narrower. In all experiments described below, the center of the aperture was always centered at the center nose poke. In other words, if the aperture was set to be $60 \mathrm{~mm}$ wide, each extrusion was positioned $30 \mathrm{~mm}$ lateral to the middle of the center nose poke. Whenever a rat poked its nose into the center nose poke, its large facial whiskers contacted the extrusions. Rats were trained to discriminate between different aperture widths with their large facial whiskers and to indicate their choice by poking their nose into either the left or right reward nose poke (below).

All aspects of the behavioral training were fully automated and computer controlled, requiring no intervention by an experimenter. Training was controlled by the Med Associates computer program MedPC and computer interface DIG700 (MED Associates, Inc., St. Albans, VT). The behavioral apparatus was enclosed in a light-proof isolation box, and the apparatus was illuminated with infrared light. The behavior of each rat was monitored via infrared-sensitive video cameras (placed above the center discrimination chamber and the outer reward chamber), thereby eliminating any need to occlude the rats' vision.

Training procedures. All rats used in these studies were Long-Evans males, weighing $\sim 350 \mathrm{gm}$ at the start of training. Two days before any training, all rats were put on a water-restriction schedule that consisted of access to water ad libitum for $1 \mathrm{hr} / \mathrm{d}$. Food was available ad libitum. Two days after the start of water restriction, behavioral training began. Rats were trained on a daily basis, each training session lasting $75 \mathrm{~min}$. At the end of each daily training session, rats were given access to water ad libitum for $1 \mathrm{hr}$. At the end of the sixth training session, rats were given access to water ad libitum for $24 \mathrm{hr}$. After this, rats were deprived of water for $24 \mathrm{hr}$, and then training was resumed. Thus, for each $7 \mathrm{~d}$ period, rats received six daily training sessions (with $1 \mathrm{hr}$ access to water after each session), followed by $1 \mathrm{~d}$ of access to water ad libitum. This restriction schedule was repeated throughout the entire course of training. All rats showed significant weight gains over the course of training, and no health problems occurred with this protocol.

Behavioral training consisted of three phases. In the first two phases, rats were trained to perform the basic procedure of the task. Phase 3 of training required rats to discriminate between different aperture widths to receive a water reward. In phase 1 , rats were trained to poke their nose into either the left or right reward nose poke to receive a $50 \mu$ l water reward. During this phase of training, the small doors in front of either the left or right reward nose pokes were randomly opened (with an intertrial interval of $30 \mathrm{sec}$ ) and remained open until rats poked their 
Table 1. Experimental overview

\begin{tabular}{lll} 
Experiment & Groups of rats & Description \\
\hline Fine-grained distance detection & One $(n=5)$ & $\begin{array}{c}\text { Quantify the ability of rats to gauge differences } \\
\text { in aperture width } \\
\text { Control experiment to ensure that rats use } \\
\text { only their large mystacial vibrissae } \\
\text { Sustematically remove individual whiskers to } \\
\text { examine multi-whisker integration and } \\
\text { whisker functionality }\end{array}$ \\
Role of whisker movement & Same group as above & $\begin{array}{c}\text { ARCS INTACT }(n=4) \\
\text { ROWS INTACT }(n=4) \\
\text { movement }\end{array}$ \\
Involvement of barrel cortex & $\mathrm{FN}^{a}$ cut $(n=3)$ & $\begin{array}{c}\text { Lesion barrel cortex to examine involvement of } \\
\text { this cortical region }\end{array}$
\end{tabular}

A brief description of each of the five different experiments and the experimental groups of rats included in each experiment.

${ }^{a}$ Facial nerve.

${ }^{b}$ Somatosensory cortex.

nose into them. After doing so, a $50 \mu$ water reward was delivered to the nose poke where the rat could easily lick it up. All rats quickly learned to poke their nose into a reward poke as soon as the door opened. The majority of rats performed over 80 trials during the first session (maximum possible number of trials, 150) and were subsequently moved on to phase 2 of training beginning with session 2 (below). All remaining rats performed $>80$ trials during session 2 and were moved to phase 2 the following day.

In phase 2 of training, rats were trained to enter the center discrimination chamber, poke their nose into the center nose poke, and then back out into the outer reward chamber and poke their nose into either the left or right nose poke to receive a water reward. During this training phase, rats were initially placed in the outer reward chamber with the center door closed. At the start of a trial, the center door was opened. Rats learned to proceed directly to the center nose poke and poke with their nose. After doing this, the small door in front of either the left or right reward nose poke (randomly chosen by computer control) was immediately opened and remained open until the rat backed out into the outer reward chamber and poked its nose into the open reward poke to receive the $50 \mu \mathrm{l}$ water reward. Immediately after poking their nose into the open reward poke, the $50 \mu \mathrm{l}$ reward was delivered, the center door was closed, and $10 \mathrm{sec}$ later, the door in front of the open reward poke was closed. A new trial began $30 \mathrm{sec}$ later. This procedure was repeated throughout the $75 \mathrm{~min}$ session. All rats received daily training sessions until they performed at least 50 trials in a single session (maximum possible number of trials per session, 112). After performing at least 50 trials per session, rats were moved to phase 3 of training (below). Rats acquired this phase of training quickly. Greater than $75 \%$ of the rats performed at least 50 trials by the end of the second session. All remaining rats performed at least 50 trials per session by the end of the third or fourth session.

Phase 3 of training was similar to phase 2 except that, in this phase, rats had to discriminate between the wide and narrow apertures to receive a water reward. Rats were initially placed in the outer reward chamber with all doors closed. Before the start of the first trial in a training session, the variable-width aperture was randomly set, by computer control, to one of two possible widths: $85 \mathrm{~mm}$ (wide) or $52 \mathrm{~mm}$ (narrow). At the start of a session, the center door was opened, and rats proceeded directly to the center nose poke to poke with their nose. As rats approached the center nose poke, their large facial whiskers contacted the walls of the variablewidth aperture (see Fig. 2). Immediately after rats poked their nose into the center nose poke, the doors in front of both left and right reward nose pokes were opened simultaneously, whether the aperture was set wide or narrow. These doors remained open until the rat backed out of the center discrimination chamber and poked its nose into either the left or right reward nose poke.

To receive a water reward, rats had to poke their nose into the "correct" reward nose poke, the left nose poke when the aperture was narrow or the right nose poke when the aperture was wide. If the rats poked their nose into the correct nose poke, a $50 \mu \mathrm{l}$ water reward was immediately delivered. If the rats poked their nose into the "incorrect" nose poke, no reward was delivered. Immediately after the rats poked their nose into either the correct or incorrect nose poke, the sliding door between the outer reward chamber and the center discrimination chamber was closed, and $10 \mathrm{sec}$ later, the doors in front of both the left and right reward nose pokes were closed. Also, the aperture was reset to wide $(85 \mathrm{~mm})$ or narrow $(52 \mathrm{~mm})$ (chosen randomly by computer control). Thirty seconds later, the next trial began when the sliding door opened, allowing access to the center discrimination chamber. Wide and narrow trial types were presented randomly throughout the $75 \mathrm{~min}$ session.

In summary, phase 3 of training required rats to enter the center discrimination chamber and poke their nose into the center nose poke. In doing so, their large facial whiskers contacted the variable-width aperture. Rats then had to back out into the reward chamber and correctly poke their nose into either the left or right nose poke to receive a water reward, the left nose poke if the aperture was narrow $(52 \mathrm{~mm})$ or the right nose poke if the aperture was wide $(85 \mathrm{~mm})$. Rats received a $50 \mu \mathrm{l}$ water reward for a correct discrimination. During the first training session of phase 3 (and all sessions of phases 1 and 2), a 2 W overhead lamp illuminated the behavioral apparatus. During the second and all subsequent training sessions of phase 3, the light was turned off. During these sessions, rats performed the task in complete darkness, thereby eliminating any visual cues.

Rats received daily training sessions with aperture settings of $85 \mathrm{~mm}$ (wide) and $52 \mathrm{~mm}$ (narrow) until they performed at least $75 \%$ of the trials correctly in three consecutive sessions. As described below (Results; see Fig. 3) rats reached this performance criterion in an average of six sessions. After the rats reached this performance criterion, the difference between the wide and narrow aperture settings was reduced a fixed amount, thereby making the discrimination more difficult (below). Rats were then trained using these new aperture settings until they again performed at least $75 \%$ of the trials correctly in three consecutive sessions. The difference between the wide and narrow settings was again reduced a fixed amount, and the rats were trained until they again reached criterion. Depending on the particular experiment, this general procedure was continued with all rats until they were performing discriminations of a desired difficulty. At this point, different manipulations were performed to test various aspects of this tactile behavior. Each of these experiments are described in detail below. Table 1 provides a brief overview of the different experiments performed.

Fine-grained distance detection. The aim of this particular experiment was to ascertain whether the vibrissal array of rats can function as a fine-grained distance detector and, if so, to quantify the limits of tactile resolution. If the vibrissal system does function as a fine-grained distance detector, then rats should be able to discriminate easily between apertures of relatively similar width. To test this, a group of rats $(n=5)$ was trained using the procedures described above. The initial aperture settings were $85 \mathrm{~mm}$ (wide) and $52 \mathrm{~mm}$ (narrow). Rats were trained until they reached criterion. On the next session, the difference between the wide and narrow settings was reduced a fixed amount (below), and rats were trained until they reached criterion. This procedure was repeated until each rat failed to reach criterion by the end of five training sessions at any particular level of difficulty. At this point, the rat was no longer trained, and the previous aperture settings (at which the rat did reach 
criterion) were considered to be the limit of that rat's tactile resolution. The following aperture settings (in $\mathrm{mm}$ ) were used during each of these steps: (1) 85 and 52, (2) 78 and 52, (3) 72 and 52, (4) 72 and 56, (5) 72 and $60,(6) 68$ and 60, (7) 68 and 62, (8) 65 and 62, and (9) 63.5 and 62.

As can be seen by the different aperture settings used in this experiment, the difference between the wide and narrow apertures was systematically reduced so that the size of the two apertures "converged" at $\sim 63$ $\mathrm{mm}$. Also, as described below, aperture settings of $60 \mathrm{~mm}$ (narrow) and $68 \mathrm{~mm}$ (wide) were routinely used in other experiments. These particular aperture settings (between 60 and $68 \mathrm{~mm}$ ) were chosen because they are approximately equivalent to the distance spanned by whiskers $\mathrm{C} 3$ or $\mathrm{C} 4$ (measured tip to tip) in the $350 \mathrm{gm}$ male rats used in these studies. Because whisker C3 is approximately in the "center" of the vibrissal array, aperture widths between 60 and $68 \mathrm{~mm}$ are approximately midway between the widest possible aperture that could be spanned by the longest whiskers (C1, D1, or straddlers) and the narrowest aperture that could be spanned by the smaller, rostralmost whiskers such as D6 or E7. In other words, aperture widths between 60 and $68 \mathrm{~mm}$ represent the approximate midpoint between the narrowest and widest distances that could be spanned by the facial whiskers of the rats used in these studies.

Cutting all whiskers. This experiment was a control experiment to ensure that the rats were using only their large facial whiskers and not the smaller whiskers around their nose and lips or other sensory cues to perform the discrimination. The same group of rats used in the previous experiment was used in this experiment. After the rats had completed the previous experiment, they were given three daily training sessions with aperture settings of $68 \mathrm{~mm}$ (wide) and $60 \mathrm{~mm}$ (narrow). As described in Results, this particular setting was only moderately difficult, and all rats performed the discrimination with a high degree of accuracy. One hour after the end of the third session (after rats had access to water for $1 \mathrm{hr})$, each rat was lightly anesthetized with halothane $(2 \%$ in oxygen), and all large facial whiskers (rows A-E and arcs 1-7 and the four straddler whiskers) on both sides of the face were cut at the level of the skin with a small pair of scissors. The fur on the face and the small whiskers around the nose and lips were not cut.

On the following day, training was continued (with the same aperture settings) to assess the effects of removing the large whiskers on the ability of rats to perform the discrimination. Rats were also trained on the following $2 \mathrm{~d}$ to test whether there was any recovery of their ability to perform the discrimination. In addition to measuring the effects on discrimination performance, several other measures were taken to ensure that whisker removal did not create any generalized changes in the behavior of the rats while they were performing the task. These measures included the following. (1) The total number of trials per session before and after whisker cuts was recorded. (2) The time between breaking the center nose poke photobeam and breaking the photobeam in front of either of the reward nose pokes was recorded. This gave a measure of the ability of the rats to navigate within the behavioral apparatus in the dark with and without facial whiskers. (3) Finally, the behavioral apparatus was illuminated with infrared light, and infrared-sensitive video cameras were used to record behavioral sessions before and after whisker cuts. These recordings were stored on videotape and analyzed off-line to compare the behavior of the rats under each condition.

Multiwhisker integration and functional similarity between whiskers. This experiment was designed to address two separate but related questions. (1) Is integration of tactual information from multiple facial whiskers necessary for rats to perform the discrimination, or can rats accurately discriminate with only one or very few whiskers per side? (2) Are different whiskers within the whiskerpad functionally equivalent, or alternatively, are individual whiskers functionally distinct subcomponents of larger functional units, for instance, whisker rows or arcs?

Briefly, the experimental approach used here was the following. Two groups of rats were trained to perform the discrimination. Then, individual whiskers were systematically removed. After each set of whisker removals, rats received three daily training sessions to assess the effects of whisker removal on discrimination performance. After these three sessions, more individual whiskers were removed, and training was continued to assess the performance of the rats with even fewer whiskers. This procedure was continued until all whiskers had been removed. Furthermore, for each of the two groups of rats, whiskers were removed in a different pattern so that, as whiskers were removed, each group had a different subset of whiskers remaining with which to perform the task. One pattern of removal preferentially left whisker rows intact, whereas the other left whisker arcs intact (see Fig. 5A; see below).

If integration of tactual input from multiple whiskers is necessary for accurate discrimination, then systematic whisker removal should result in a gradual decrease in performance that is proportional to the number of whiskers removed. In contrast, if tactual input from each whisker is processed relatively independently, then removing whiskers not specifically involved in contacting either the wide or narrow aperture should have little or no effect on performance, whereas cutting whiskers directly involved in contacting either the wide or narrow aperture should cause a sudden and sharp decrease in performance. Furthermore, if whiskers are functionally equivalent, then decreases in performance should be independent of which whiskers were cut. In contrast, if whiskers are functionally distinct and operating together with other whiskers as specific functional units, for instance, intact rows or arcs, then removing whiskers in the patterns that preferentially leave rows intact or arcs intact should have different effects on performance.

To test these issues, two groups of rats [ARCS INTACT $(n=4)$ and ROWS INTACT $(n=4)$ ] were trained until they reached criterion with aperture settings of 60 and $68 \mathrm{~mm}$; at which point each rat received five daily training sessions using these settings (as described above). After the fifth session, all rats in both groups were lightly anesthetized with halothane, and the following whiskers were cut at the skin on both sides of the face: (1) all whiskers in row A, (2) all four straddler whiskers, and (3) all whiskers in arcs 5-7. The small whiskers around the nose and lips were not cut. This left a grid of 16 intact large whiskers on each side of the face: rows B-E and arcs 1-4 (see Fig. 5A). Beginning the next day, all rats received three daily training sessions to assess the effects of these whisker cuts. After the end of the third session, another set of whiskers was cut (all previously cut whiskers were also trimmed back), and beginning the next day, all rats received three more daily training sessions to assess the effects of these whisker cuts on performance. This general procedure (selective whisker cut followed by three training sessions) was repeated until all of the whiskers had been cut.

In each of the two groups, different whiskers were cut at each of these steps. The precise pattern of whisker removal for each group is depicted graphically (see Fig. $5 A$ ). For rats in the ROWS INTACT group, whiskers were removed in a pattern that preferentially left whisker rows intact. The following whiskers were cut at each step: (1) all whiskers in row A and arcs 5-7 and the four straddler whiskers (described above), (2) whiskers in row $\mathrm{B},(3)$ whiskers in row $\mathrm{E},(4)$ whiskers in row $\mathrm{D},(5)$ whiskers $\mathrm{C} 1$ and $\mathrm{C} 4,(6)$ whisker $\mathrm{C} 2$, and (7) whisker $\mathrm{C} 3$. For rats in the ARCS INTACT group, whiskers were removed in a pattern that left whisker arcs intact. The following whiskers were cut: (1) all whiskers in row $\mathrm{A}$ and arcs 5-7 and the four straddler whiskers, (2) whiskers in arc 1, (3) whiskers in arc 4, (4) whiskers in arc 2, (5) whiskers B3 and E3, (6) whisker D3, and (7) whisker C3. As described above, each rat received three daily training sessions after each of these whisker removals to assess their ability to discriminate aperture widths with a decreasing set of intact whiskers.

Role of whisker movement during discrimination. As described in Results, video analysis of rats performing the discrimination indicated that they did not actively move their whiskers as they sampled the aperture (see Fig. 2). Thus, this experiment was intended to confirm that active movement of the whiskers was not necessary to discriminate accurately between apertures of different width. To test this, the facial nerve was cut bilaterally in a group of rats $(n=5)$ trained to discriminate between aperture widths of 60 and $68 \mathrm{~mm}$ (as described above). The nerve cut prevented any movement of the facial whiskers. Training was continued after the nerve cut, and discrimination performance before and after nerve cuts was compared.

The nerve cut procedure was as follows. After the fifth training session with aperture settings of 60 and $68 \mathrm{~mm}$, rats were given access to food and water ad libitum for $4 \mathrm{~d}$ with no training. Rats were then anesthetized with ketamine $(100 \mathrm{mg} / \mathrm{kg})$ and xylazine $(10 \mathrm{mg} / \mathrm{kg})$. The facial fur posterior to the whiskerpad was shaved. A small incision $(\sim 3 \mathrm{~mm})$ was made in the skin $\sim 3-4 \mathrm{~mm}$ posterior to whisker E1. The incision was centered over the region where the anterior parts of the ramus buccolabialis superior and ramus buccolabialis inferior branches of the facial nerve join in a short trunk before bifurcating throughout the whiskerpad [see Dörfl (1985), his Fig. 1]. The soft tissue below the skin was carefully dissected to expose this trunk of the facial nerve. To ensure that the proper portion of the facial nerve had been isolated, small pulses of current $(\sim 100 \mu \mathrm{A} / 1 \mathrm{msec})$ were applied to the nerve at this location through a small, bipolar stimulating electrode. This resulted in a clear, punctate movement of the whiskerpad. Two small loops of 7-0 surgical suture were then tied tightly around the nerve, $\sim 1 \mathrm{~mm}$ apart. The nerve was then cut between the two suture loops, and each cut end was crushed 
with a small hemostat. The wound was then sutured closed, and the same procedure was repeated on the opposite side of the face. Rats were given $5 \mathrm{~d}$ of postsurgical recovery with access to food and water ad libitum. Rats were then put on the water-restriction schedule for $2 \mathrm{~d}$. Beginning on day 8 after surgery, rats received three daily training sessions using the same aperture settings. Performance before and after facial nerve cuts was compared to assess the ability of the rats to perform the discrimination without active whisker movements.

Involvement of barrel cortex. This experiment was designed to test whether the barrel region of the primary somatosensory cortex (SI) is necessary for rats to perform these tactile discriminations. A group of rats $(n=3)$ was trained to perform the discrimination (as described above) until each rat reached performance criterion with aperture settings of $68 \mathrm{~mm}$ (wide) and $60 \mathrm{~mm}$ (narrow). Each rat then received five daily training sessions with these aperture settings. After the fifth session, the barrel cortex was aspirated bilaterally. After $7 \mathrm{~d}$ of postsurgical recovery, training was continued (using the same aperture settings as before surgery) to determine whether the barrel cortex is critically involved in performance of this task.

The cortical aspiration lesions were performed as follows. After the last training session with aperture settings of 60 and $68 \mathrm{~mm}$, the rats were given access to water and food ad libitum for $4 \mathrm{~d}$ (with no training sessions). On the following day, rats were anesthetized with ketamine $(100 \mathrm{mg} / \mathrm{kg})$ and xylazine $(10 \mathrm{mg} / \mathrm{kg})$ and placed in a stereotaxic head holder. The scalp was incised, and the skin and periostium over the region of the barrel cortices were retracted. A craniotomy $(\sim 5 \mathrm{~mm}$ diameter) was made over the region of each barrel cortex, centered $\sim 3$ $\mathrm{mm}$ posterior and $4.5 \mathrm{~mm}$ lateral to the bregma skull suture. A tungsten microelectrode $(Z \cong 0.5 \mathrm{M} \Omega$ at $1000 \mathrm{~Hz}$ ) was lowered $\sim 0.7 \mathrm{~mm}$ into several locations within the region of the barrel cortex. Neural activity recorded by the electrode was amplified and monitored on an audio amplifier while the contralateral large facial whiskers were manually stimulated. This procedure to locate the boundaries of the barrel region was used to facilitate accurate lesioning of the barrel cortex. After this mapping procedure, the dura overlying the barrel cortex was retracted, and the underlying barrel cortex was aspirated. An electrocauterizer was used to stanch bleeding. After the aspiration was complete, the lesion site was filled with Gelfoam soaked in saline. A layer of bone wax was placed over the lesion in place of the removed skull. A thin layer of dental acrylic was placed over the bone wax to seal and protect the lesion site securely. A similar lesion was then performed on the opposite barrel cortex. Finally, the skin over the skull was sutured closed. The rats were then given $5 \mathrm{~d}$ of postsurgical recovery with access to food and water ad libitum. On days 6 and 7 after surgery, rats were again put on the water-restriction schedule in which water was available for only $1 \mathrm{hr} / \mathrm{d}$. On day 8 , daily training sessions resumed using the same aperture settings and procedures as before surgery. Rats were given three daily training sessions to assess the effects of the cortical lesions.

Three other rats from a different study were used as controls. These rats were trained using procedures identical to those used on the rats that received SI aspirations. The surgery on these rats was identical except that, instead of lesioning the SI cortex, a recording array of eight microwire electrodes with an infusion cannula was chronically implanted in each barrel cortex [surgical procedures have been described in detail elsewhere (Krupa et al., 1999)]. After surgical recovery, training was continued (as with the lesioned rats), and performance before and after the implant was compared.

After all training, all rats were injected with a lethal dose of pentobarbital and then perfused with saline and $10 \%$ formalin. The brains were removed, fixed in formalin-sucrose, sectioned, and stained with cresyl violet and Prussian blue (rats implanted with cannula and recording electrodes). For the three rats with the aspiration lesions, the extent of the lesions was assessed and plotted on standard sections (Paxinos and Watson, 1986). For the three rats with infusion cannula and recording electrodes, placement of the cannulas and microwires was histologically confirmed.

\section{RESULTS}

\section{Rate of acquisition and general behavioral characteristics}

As described above, rats typically acquired the first phase of training (simply poking their noses into either the left or right reward nose pokes for a water reward) within two daily training sessions. The majority of rats acquired phase 2 of training within two to three sessions. Finally (see Fig. 3), rats required an average of six daily sessions to reach the learning criterion of $75 \%$ correct in three consecutive sessions after they began phase 3 of training with aperture settings of 85 and $52 \mathrm{~mm}$. Thus, the typical rat required a total of 10 daily training sessions to progress from completely naive to performing the discrimination accurately. Rats typically performed $\sim 80$ or more trials during a training session.

One of the primary goals during the development of this behavioral task was to design the training apparatus (Fig. 1) so that freely behaving rats sampled the variable-width aperture, using only their large facial whiskers, in a highly consistent, very repeatable, stereotypical manner. Figure 2 is a series of video frame captures that show an individual rat approaching, contacting, and withdrawing from both a wide $(68 \mathrm{~mm})$ and narrow (62 $\mathrm{mm}$ ) aperture. As can be seen, the rat approaches and samples the aperture in virtually identical manners from trial to trial. This was true from trial to trial within a session as well as from session to session. Furthermore, there was remarkably little difference in the manner in which different rats approached and sampled the aperture. The behavior of rats as they performed the task was visually monitored by viewing their activity via the infrared video cameras located above the center discrimination chamber and outer reward chamber (Fig. 2).

During the discriminations, all rats followed the same behavior seen in Figure 2; rats rapidly proceeded straight to the center nose poke while their large facial whiskers contacted the aperture walls. After breaking the photobeam in the center nose poke, the rats backed out of the center discrimination chamber, turning left or right toward the appropriate reward poke. Notice that only the large facial whiskers contact the aperture. We did not observe any rats using alternative strategies to solve the discrimination, for instance, moving their head side to side so that the small whiskers around their nose and lips or other parts of their face could contact the aperture walls. The consistency with which rats contacted the aperture with their large facial whiskers was critically important for the experiments in which different whiskers were removed. If individual rats sampled the aperture in different ways using different sets of whiskers, for instance, the smaller whiskers around the nose and lips, comparison of the behavioral results after whisker removal would be impossible.

\section{Fine-grained distance detection}

The purpose of this experiment was to determine whether the vibrissal array of the rat could function as a fine-grained distance detector and, if so, to gain some insight into the limits of resolution. The results are shown in Figure 3. Rats rapidly learned to accurately discriminate between very small differences in aperture widths. Of the five rats in this experiment, all five reached criterion in five sessions with aperture settings of $62 \mathrm{~mm}$ (narrow) and $68 \mathrm{~mm}$ (wide). Three of the five rats reached criterion within five sessions with aperture settings of 62 and $65 \mathrm{~mm}$. At these settings, one other rat performed $>75 \%$ on two but not three sessions. Finally, the last rat performed near chance levels at these aperture settings. All rats performed near chance levels with aperture settings of 62 and $63.5 \mathrm{~mm}$. Thus, aperture settings between 62 and $68 \mathrm{~mm}$ and 62 and $65 \mathrm{~mm}$ appear to be near the limit of the resolution of the rats. Apertures of 62 and $68 \mathrm{~mm}$ represent a difference of $9 \%$, and apertures of 62 and $65 \mathrm{~mm}$ are $5 \%$ different.

As seen in Figure 2, rats were capable of performing the discrimination very rapidly. Their large facial whiskers remained 
Narrow Aperture
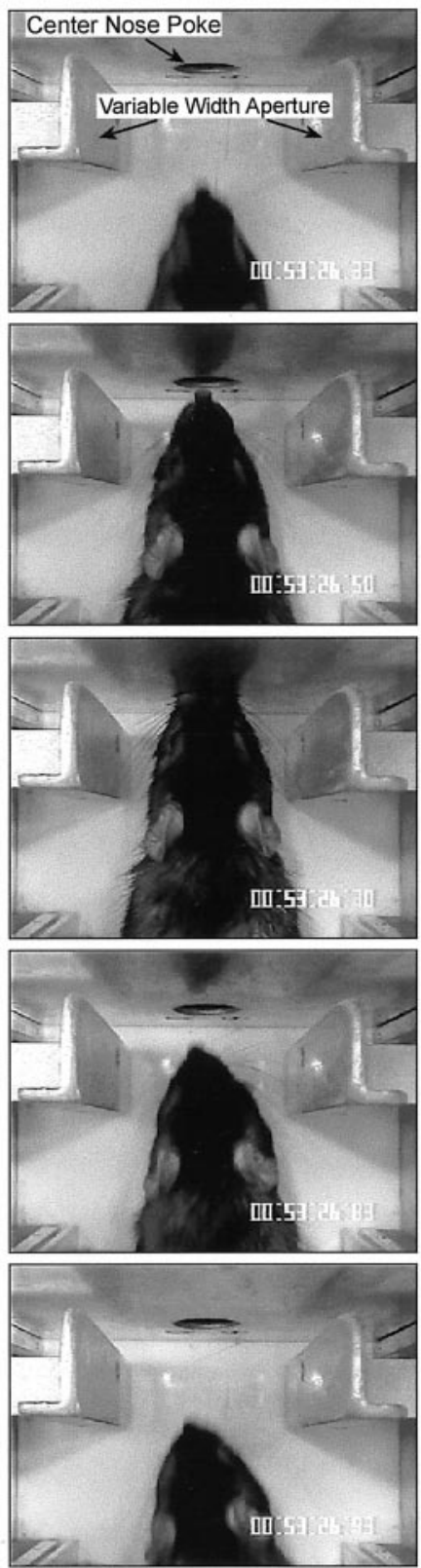

Wide Aperture
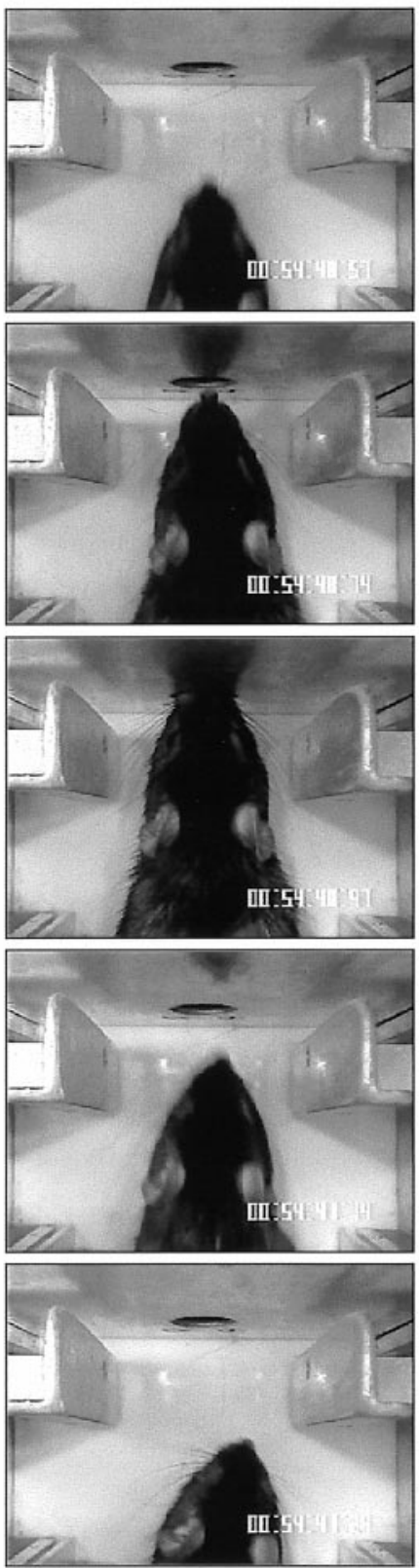

Figure 2. Each column represents a series of still frames (captured from videotape) showing a rat performing a correct discrimination of a narrow (62 $\mathrm{mm}$; left column) or wide (68 mm; right column) aperture. The video sequence progresses from top to bottom. The topmost frame in each column shows the rat approaching the center nose poke and variablewidth aperture. The second frame shows the whiskers in contact with the aperture. The third frame shows the rat completing the center poke. The fourth and fifth frames show the rat withdrawing from the center poke and aperture. In these two frames it can be seen that the rat has begun to turn left (narrow) or right (wide), indicating that the rat has correctly determined the relative width of the aperture and is turning toward the appropriate reward poke. Also, only the large facial whiskers contact the aperture walls. The smaller whiskers around the nose and lips do not contact the aperture. Finally, notice the similarity between the wide and narrow apertures. Even when shown side-by-side, it is difficult to distinguish between the two visually. Rats were capable of discriminating apertures even more similar in width $(62$ and $65 \mathrm{~mm})$. The time display in the lower right-hand corner of each frame (hours:minutes:seconds:hundredths of a second) shows the elapsed time between each frame. The chamber is illuminated with an infrared light source so that the rat cannot use visual cues.

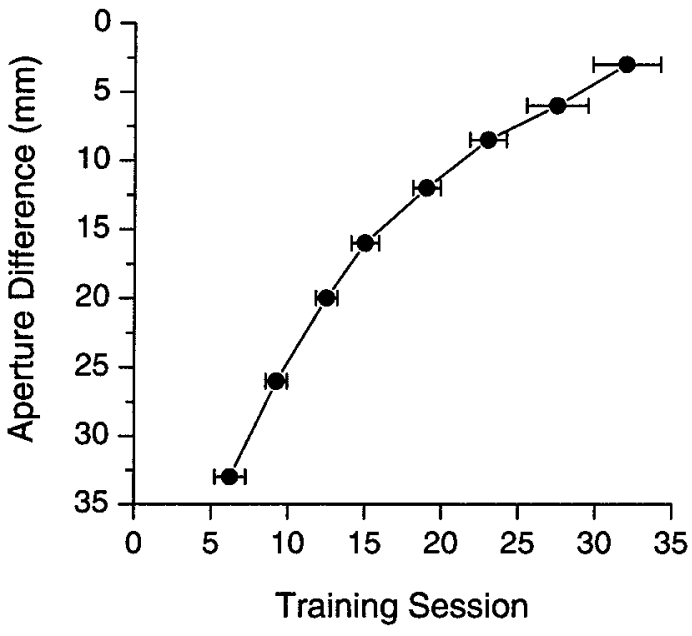

Figure 3. Mean $( \pm$ SEM) cumulative number of training sessions (during phase 3 training) necessary for a group of rats $(n=5)$ to reach criterion at each progressively more difficult aperture setting. Each data point represents a different step in the sequence (see Materials and Methods, Fine-grained distance detection). The first data point (Aperture Difference of $33 \mathrm{~mm}$ ) corresponds to aperture settings of $85 \mathrm{~mm}$ (wide) and $52 \mathrm{~mm}$ (narrow). The final data point (Aperture Difference of $3 \mathrm{~mm}$ ) corresponds to aperture settings of 62 and $65 \mathrm{~mm}$. Rats rapidly learn to perform the discrimination, and they are capable of accurately discriminating between apertures that are very similar in width.

in contact with the aperture very briefly. The mean time from when the whiskers of the rats first contacted the aperture until their nose broke the center nose poke beam was $290 \pm 15 \mathrm{msec}$ (based on video analysis of five rats performing discriminations between apertures of 62 and $68 \mathrm{~mm}$ ). Also, as seen in Figure 2, rats began turning left or right almost immediately after they began to withdraw from the center nose poke, indicating that they had decided whether an aperture was wide or narrow by the time they had completed the center nose poke. Furthermore, rats were capable of performing the discrimination even more rapidly if they were not required to break the center nose poke beam but, instead, were simply required to sample the aperture to receive a water reward (data not shown). Moreover, rats almost never sampled an aperture more than once per trial. In short, rats were able to gauge aperture widths accurately with only the briefest contact with the aperture. Just a short touch was sufficient for accurate discriminations.

Slow motion and frame-by-frame video analysis of the whiskers of the rats as they sampled the aperture during a discrimination revealed that rats did not actively "whisk" the aperture. Instead, their whiskers were held relatively still as they approached and sampled the aperture. As rats entered the center discrimination chamber, their whiskers were typically positioned so that the rostralmost whiskers were pointed almost directly forward. The whiskers slightly caudal to these were angled in the forward direction, but somewhat to the side. Whiskers in the middle of the vibrissal array typically pointed almost directly sideways, perpendicular to the faces of the rats. The more caudal whiskers were angled rearward, with the most caudal whiskers angled the most rearward. Thus, when held in this position, the vibrissal array formed almost a hemisphere around each side of the face of the rat. The whiskers were held in this position until they contacted the aperture, at which point they were deflected rearward by the walls of the aperture as the rat entered the aperture. The angle of deflection (relative to the original position of the whisker) de- 


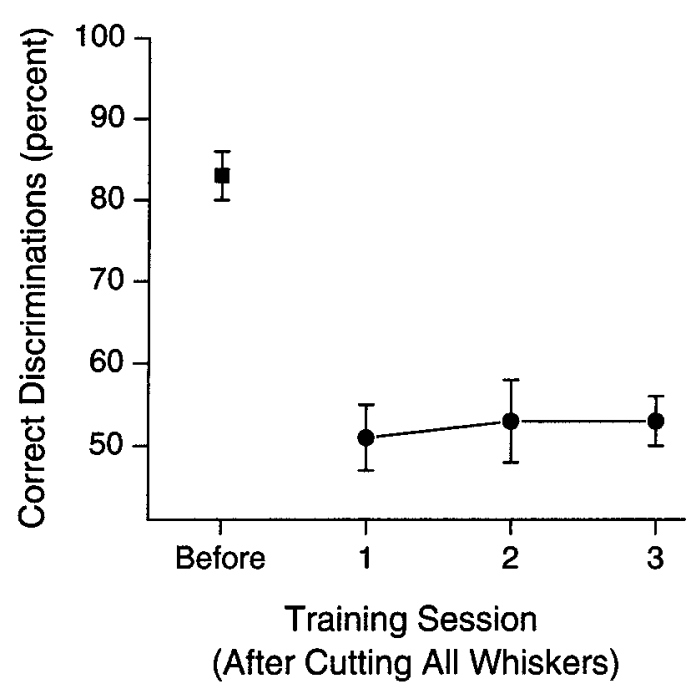

Figure 4. Mean $( \pm$ SEM) percentage of correct discriminations for a group of rats $(n=5)$ in the session before cutting all of the large facial whiskers on both sides of the face (Before) and for each of the three training sessions (1-3) after the whisker cuts. Cutting all large facial whiskers completely abolished the ability of the rats to perform the discrimination. There was no recovery of performance over the three sessions after whisker cuts.

pended on the particular whisker deflected and the width of the aperture. For instance, the more rostral whiskers contacted the aperture at a point along their shaft nearer the outer end where the whisker is very fine. Because the shaft of these whiskers is very fine at the point of contact with the aperture, only the end of the whisker was deflected rearward. The thicker portion of the whisker shaft nearer the skin was deflected only slightly. In contrast, the longer, more caudal whiskers contacted the aperture walls at points along their shaft where the whisker was much thicker and, hence, stiffer. When these whiskers contacted the aperture walls, almost the entire whisker was deflected rearward, as if deflecting a semirigid lever that pivots at the skin. Of course, depending on the width of a particular aperture setting, the degree to which any particular whisker was deflected only at the tip or, instead, more along the entire shaft varied from trial to trial.

\section{Cutting all whiskers}

This experiment was conducted to ensure that rats were using only their large facial whiskers and not other sensory cues such as the smaller facial whiskers around the nose and lip to solve the discrimination. The results are shown in Figure 4. Cutting the large facial whiskers (whiskers in rows A-E and arcs 0-7) on both sides of the face completely abolished the ability of rats to discriminate accurately between apertures of 60 and $68 \mathrm{~mm}$, an aperture difference of only moderate difficulty. The mean performance of the rats after the whisker cut was significantly reduced from $83.1 \pm 1 \%($ mean $\pm \mathrm{SEM})$ to chance performance $[50.1 \pm$ $4 \% ; t(4)=11.01 ; p<0.0005]$. No recovery was seen over $3 \mathrm{~d}$ of postcut training $\left(F_{(2,12)}<1\right)$. Importantly, this effect was limited only to the ability of the rats to discriminate aperture width. No other observable aspect of behavior was affected. There was no difference between the mean number of trials performed (per session) with and without whiskers [mean \pm SEM, $73 \pm 6$ vs $71 \pm$ $5 ; t(4)=0.16 ; p>0.05]$. The mean time (in seconds) between breaking the center nose poke beam and breaking either of the reward nose poke beams also was not different $[1.32 \pm 0.13$ vs
$1.37 \pm 0.15 ; t(4)=-0.35 ; p>0.05]$, indicating that cutting the facial whiskers did not affect the ability of the rats to navigate and move around within the behavioral apparatus. Finally, comparison of the behavior of the rats recorded on videotape from sessions before and after cutting the facial whiskers revealed no observable differences in behavior.

\section{Multiwhisker integration and functional similarity between whiskers}

The purpose of this experiment was to determine the degree to which tactile input from multiple whiskers is integrated to discriminate accurately between wide $(68 \mathrm{~mm})$ and narrow $(60 \mathrm{~mm})$ apertures and to determine the degree to which individual whiskers within the whiskerpad are functionally equivalent or functionally distinct. The results are shown in Figure 5. Figure $5 A$ graphically summarizes the patterns of whisker removals for both groups of rats in this experiment.

Figure $5 B$ shows the overall effect of removing individual whiskers on performance of the discrimination. Each data point in Figure $5 B$ represents the average performance of all rats (groups ROWS INTACT and ARCS INTACT) during the three sessions after each set of whisker removals. There is a significant decline in performance with removal of whiskers $\left(F_{(7,49)}=41.8 ; p<0.0001\right)$. This gradual decrease in performance is highly correlated $[r(6)=0.95 ; p<0.001]$ with the decrease in total number of whiskers remaining on each side of the face. Accurate performance of the discrimination requires a relatively large number of intact whiskers, $\sim 12$ or more on each side of the face. These results indicate that accurate discrimination requires integration of tactile input from many individual whiskers.

Figure $5 C$ shows the effects of whisker removal for each group of rats. There was no difference between the ROWS INTACT and ARCS INTACT groups $\left(F_{(1,6)}=0.16 ; p>0.05\right)$ and no significant interaction of group and number of remaining whiskers $\left(F_{(7,42)}=1.4 ; p>0.05\right)$. The performance of each group decreased at the same rate despite removing different sets of whiskers. These results indicate that different whiskers within the vibrissal array are functionally equivalent because the different whiskers contribute equally to accurate discriminations. For instance, when each group of rats had only four whiskers remaining on each side of the face, the ROWS INTACT group had row $\mathrm{C}$ intact, whereas the ARCS INTACT group had arc 3 intact. Thus, there was only a single whisker (C3) in common between groups. Despite these differences in the complement of intact whiskers, the performance of each group did not differ significantly.

Although the results of this experiment indicate that tactile input from many whiskers must be integrated to perform the discrimination, the precise nature of the tactual information derived from these whiskers remains unclear. For instance, by exploiting the fact that whisker length varies in a highly conserved manner, a rat could potentially gauge the width of an aperture by simply sensing which particular whiskers were touched or untouched as whiskers contacted the aperture, as suggested by Brecht et al. (1997). It is quite possible that rats used a simple touched/untouched tactile signal to perform the discrimination when the aperture settings were quite different. However, in this experiment, the aperture settings (60 and $68 \mathrm{~mm}$ ) were such that, when 12 or fewer whiskers remained, all whiskers contacted the aperture walls at both settings. However, when rats had 12 or 8 whiskers remaining per side, they were still able to perform the task at reasonably high levels of accuracy, although all whiskers 
A

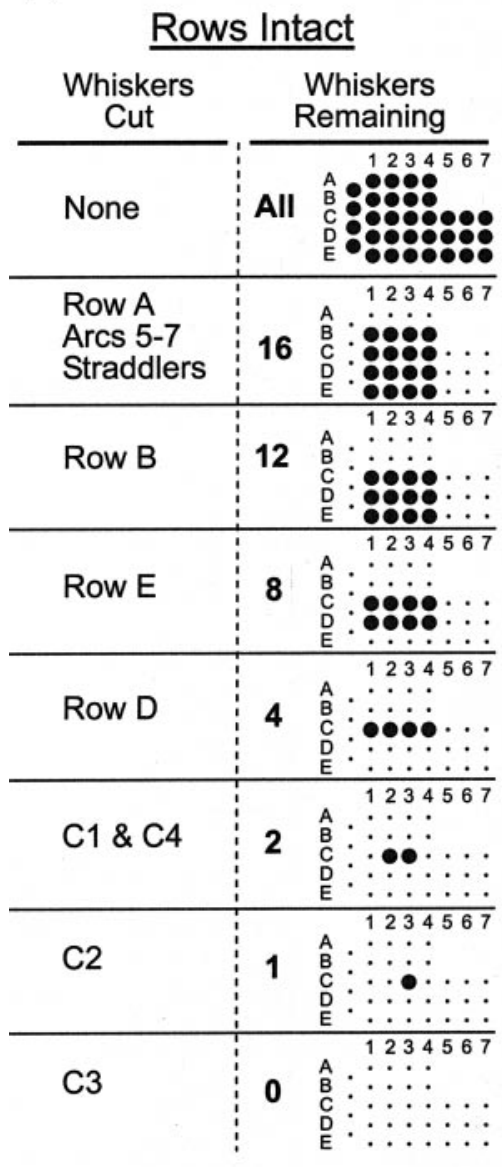

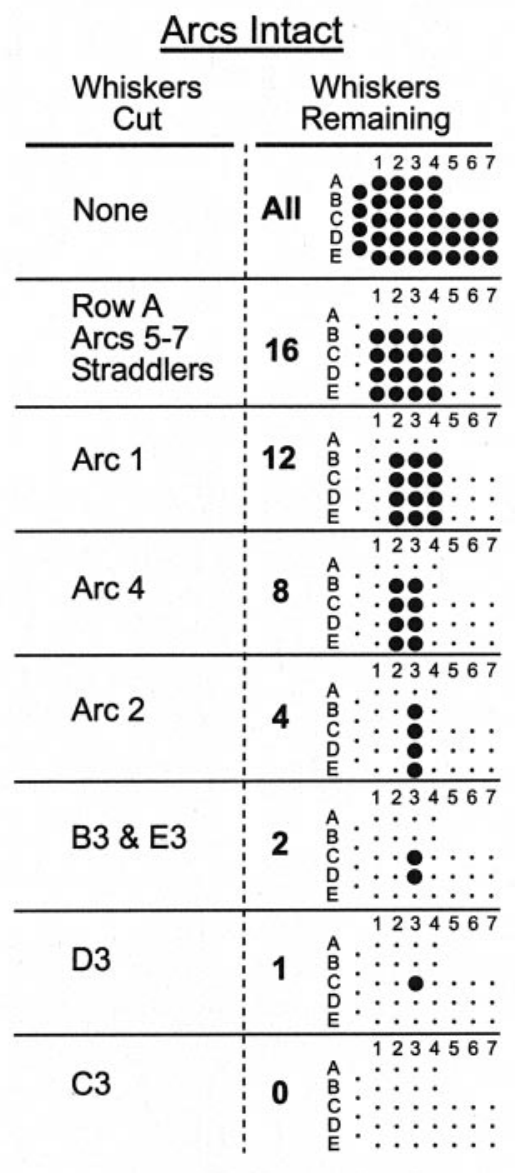

B
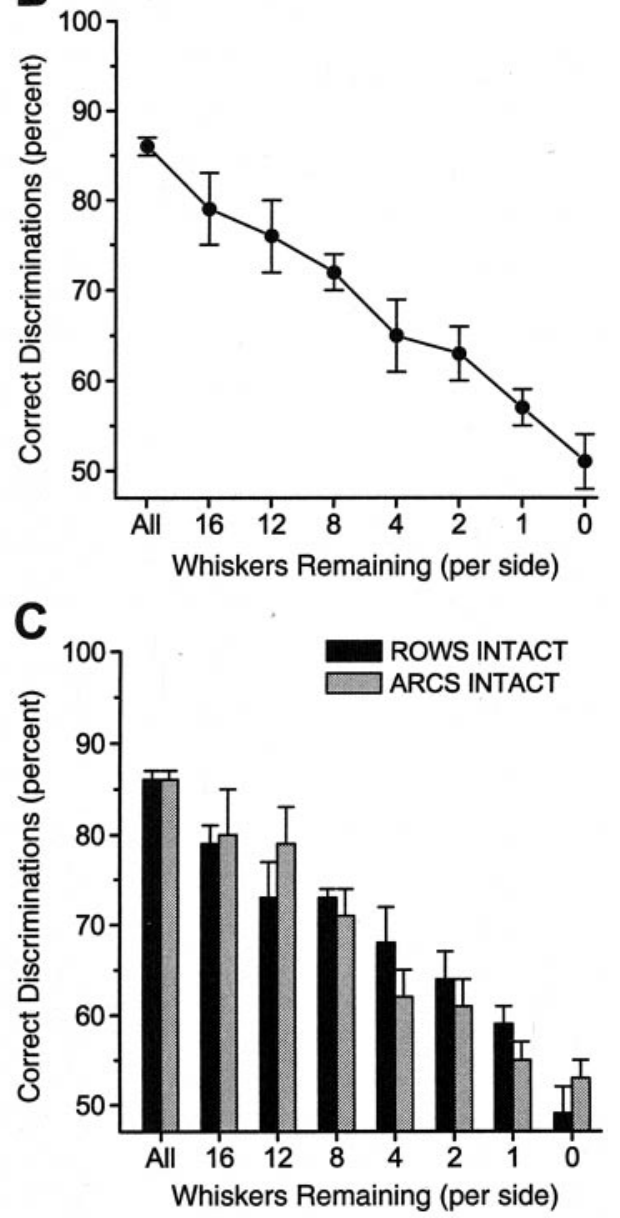

Figure 5. A, Graphical depiction of the whiskers removed at each stage of the experiment. The leftmost two columns refer to the ROWS INTACT group, and the rightmost two columns refer to the ARCS INTACT group. The Whiskers Cut columns describe which whiskers were cut at each stage. The Whiskers Remaining columns show how many total whiskers were remaining and a depiction of which whiskers. Each grid represents the vibrissal array. Large dots represent intact whiskers; small dots are cut whiskers. Thus, after the first set of whisker cuts, a grid of 16 intact whiskers remained (Rows $B-E$, Arcs 1-4). Subsequent cuts further reduced the total number of intact whiskers. As seen in each group, different sets of whiskers were removed at each step. $B$, Mean $( \pm$ SEM) percentage of correct discriminations for all rats $(n=8)$ in which whiskers were systematically removed. There is a gradual decrease in performance that is correlated with the total number of whiskers removed. Each data point represents the mean percentage of correct discriminations of all three training sessions for both groups of rats (ROWS INTACT and ARCS INTACT) after each set of whisker cuts. $C$, Discrimination performance for each separate group of rats in which different sets of whiskers were cut. There is no difference between groups, indicating that different whiskers in the vibrissal array are functionally equivalent.

contacted the aperture walls on both wide and narrow trials. Thus, in this experiment, rats would not be able to gauge aperture width simply by decoding which particular whisker(s) was touched or untouched at each aperture setting. Furthermore, in the previous experiment that examined fine-grained distance detection, rats were capable of discriminating between apertures that differed by a total of $3 \mathrm{~mm}$. In this case the position of each wall of the aperture differed by only $1.5 \mathrm{~mm}$. To discriminate between these distances using only a touched/untouched signal, the position of the vibrissae of the rats from trial to trial could not differ by $>1.5 \mathrm{~mm}$; otherwise, the complement of touched/untouched whiskers would also differ from trial to trial. Although rats sampled the aperture in a very consistent manner, it is unlikely that whisker positions remained this consistent from trial to trial. Together, these results indicate that, although a binary touched/ untouched signal may be sufficient to perform discriminations when the apertures are fairly different, more complex tactual information from individual whiskers must be integrated by the trigeminal system to discriminate between similar apertures.
Possible tactual cues might include the following. As rats enter the aperture, the facial whiskers are deflected rearward. The magnitude of this deflection and the resultant deflection velocity would vary with different apertures, and this difference might be detected. Electrophysiological recording studies show that somatosensory neurons throughout the trigeminal system respond differently to small differences in either the amplitude or velocity of single-whisker deflections (Zucker and Welker, 1969; Waite, 1973; Simons, 1978; Ito, 1981, 1985; Peterson et al., 1998; Pinto et al., 2000). Alternatively, as the whiskers encounter different apertures, the position along the whisker shaft where the aperture wall first contacts the whisker will differ: closer to the base with narrow apertures and closer to the tip with wider apertures. The diameter of individual whiskers tapers along the length of the whisker, thereby altering the mechanical properties (e.g., flexibility or stiffness) of the whisker along the shaft. Thus, contacting the whisker at different points along the shaft would likely cause different tactual signals to be transduced at the base of the whisker. Another possibility is that the precise temporal sequence 


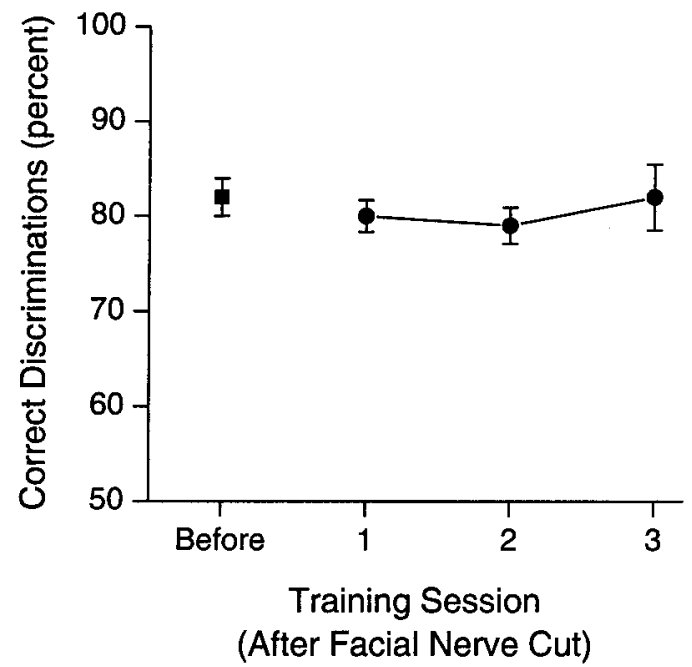

Figure 6. Mean $( \pm$ SEM) percentage of correct discriminations for a group of rats $(n=3)$ in the session before bilaterally cutting the facial nerve (Before) and for each of the three training sessions (1-3) after the nerve cut. Cutting the facial nerve did not affect performance.

with which individual whiskers contact different apertures varies depending on the width of the aperture. The different timings of these whisker contacts might be detected. Electrophysiological recordings of neurons in barrel cortex show that stimulating multiple whiskers in different temporal sequences can significantly alter responses compared with responses to single-whisker stimuli (Simons, 1985). At present, these possibilities remain speculative. Further experimentation will be necessary to elucidate the precise nature of the tactual information that is integrated to gauge aperture width.

\section{Role of whisker movement during discrimination}

Of the five rats that initially underwent facial nerve cut surgery, two displayed some signs of whisker movements on at least one side of the face after surgical recovery, indicating that the facial nerve cuts in these animals were not complete. Because whisker movements in these two rats were not completely abolished, these rats were excluded from further analysis. In the remaining three rats, whisker movements on both sides of the face were completely abolished after the facial nerve cuts. There were no observable spontaneous whisker movements, nor were there any movements after novel stimuli were placed in the home cages of the rats [intact rats universally approach and actively sniff novel objects, resulting in vigorous movements of the facial whiskers (Welker, 1964)]. All other observable behaviors appeared normal in these three rats.

The effects of facial nerve cuts on the ability to discriminate between aperture widths of 68 and $60 \mathrm{~mm}$ are shown in Figure 6. There was no significant effect on discrimination; the performance of the rats remained the same both before and after the facial nerve cuts $[t(2)=0.75 ; p=0.53]$. No significant change in performance was observed over 3 d of retraining $\left(F_{(2,4)}=0.26\right.$; $p=0.78)$. As stated above, video analysis of normal rats performing the task indicated that rats performed the discrimination without any significant movement of their facial whiskers. Slow motion and frame-by-frame video analysis was used to examine whisker biometrics of the facial nerve cut rats performing the discrimination before and after the nerve cut. Although the rats with facial nerve cuts could not move their whiskers, their whis- kers did remain extended outward from the face in a pattern that was similar to pre-nerve cut behavior. As such, their whiskers did contact the aperture walls in a way that was similar to that of normal rats. Also, it should be noted that although both normal rats and facial nerve cut rats did not move their whiskers during the discriminations, the rats themselves did move into and away from the aperture. This, of course, did result in a relative movement of the whiskers across the aperture. The mean time from when the whiskers first contacted the aperture until the time the rats entered the center nose poke was determined during the training session before and after the nerve cut. This gives a measure of the mean velocity of the rat's head as it enters and samples the aperture. There was no difference in this time before and after the nerve cut (mean \pm SEM, $0.28 \pm 0.03$ and $0.29 \pm$ $0.02 \mathrm{sec}$, respectively; $\left.F_{(1,4)}=0.12 ; p>0.05\right)$, indicating that the relative velocity in which the whiskers moved across the aperture did not change after nerve cuts. However, there was never any evidence that rats used a "whisking" motion (i.e., rapid and repetitive protraction and retraction of the whiskers) to sample the aperture or to perform any other aspect of the task.

\section{Involvement of barrel cortex}

Bilateral lesions of the barrel region of the SI cortex significantly impaired the ability of rats to perform the discrimination accurately (Tukey $\mathrm{HSD}, p=0.01$ ), reducing performance to near chance levels (Fig. 7A). In Figure $7 A$, the performance of each of the cortically lesioned rats is shown for each training session. The performance of control rats did not differ before and after the surgical procedure (Tukey HSD, $p=0.67$ ). There was no significant recovery of performance over the three postlesion training sessions $\left(F_{(2,4)}=0.38 ; p=0.7\right)$. As with cutting all of the facial whiskers (above), there were no other observable effects on the behavior of the rats before or after the lesion. Thus, the barrel region of the SI cortex is critically involved in accurate performance of the discrimination. This result is consistent with previous lesion studies that have shown a critical role for the SI cortex in the performance of other, whisker-dependent behaviors (Hutson and Masterton, 1986; Hurwitz et al., 1990; Guic-Robles et al., 1992).

Additionally, in a separate study, we reversibly inactivated the barrel region of the SI cortex with microinjections of the $\mathrm{GABA}_{\mathrm{A}}$ agonist muscimol (Shuler et al., 2000) as rats performed aperture discriminations. Reversible inactivation of the SI cortex completely abolished the ability of the rats to perform the discrimination with no effect on other aspects of task behavior. Performance returned to preinactivation levels on subsequent sessions when no muscimol was injected or when saline control injections were administered. Together, these reversible inactivation results as well as the permanent lesion results indicate that the performance decreases after either reversible or permanent lesions of the SI cortex result from disruption of sensory processing that is mediated by this cortical region and not an indirect or generalized result of brain injury. Although these lesion results clearly indicate that the barrel cortex plays a critical role in accurate aperture discrimination, they do not address the issue of whether this cortical area is essential for accurate performance. In other words, could cortically lesioned rats, after extensive postlesion training, reacquire the ability to discriminate, or would their performance be permanently impaired? Further experimentation will be required to address this issue.

Figure $7 B$ shows the extent of each lesion on standard sections. 

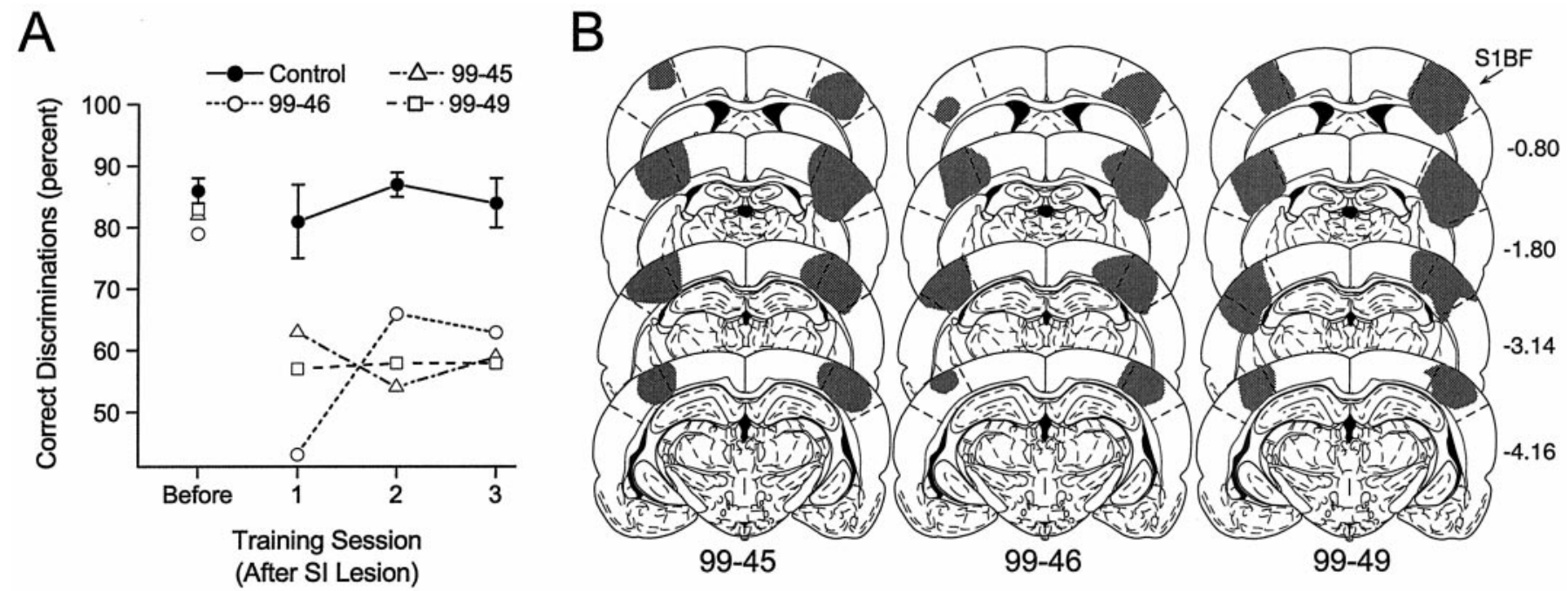

Figure 7. A, Mean $( \pm$ SEM) percentage of correct discriminations in the session before aspiration of the barrel region of the SI cortex (Before) and for the three sessions (1-3) after cortical lesions for each of the rats with SI lesions $(n=3$; open symbols). Cortical lesions significantly impaired the ability of the rats to perform the discrimination. The performance of control rats $(n=3$; Control $)$ did not differ before or after surgery. $B$, Extent of the cortical lesions (gray regions, shown on standard sections) for each cortically lesioned rat. Numerals on the right represent the distance (millimeters) of each section posterior from the bregma skull suture. Regions demarcated by dashed lines represent the barrel region of the primary SI cortex. Atlas templates are modified from Paxinos and Watson (1986). S1BF, Barrel region of the SI cortex. Numerals below each column identify individual rats.

Lesions are generally restricted to the region of the barrel cortex with little overlap into neighboring areas of cortex.

\section{DISCUSSION}

The mystacial vibrissae/trigeminal somatosensory system of the rat is a widely used model for studying the properties of somatosensation in the mammalian brain (for review, see Waite and Tracey, 1995). Numerous electrophysiological studies of this system have provided detailed insights into mechanisms of tactile processing at the single-neuron level (for review, see Simons, 1995; Moore et al., 1999; Ghazanfar and Nicolelis, 2001). In contrast, much less is known about properties related to acquisition and processing of behaviorally relevant tactual information in the awake behaving rat. Here, we examined several issues associated with different functional aspects of the vibrissal array in rats actively performing tactile discriminations.

First, we examined the basic functional nature of the vibrissal array itself. Previous studies have shown that the vibrissal array of the rat may function as a skin-like receptive sheet used to detect or discriminate the texture of different surfaces (Guic-Robles et al., 1989; Carvell and Simons, 1990). In contrast, a recent study challenged this hypothesis, suggesting instead that the large mystacial vibrissae act as discrete, punctate tactile sensors that serve to detect differences in distance to nearby objects or surfaces (Brecht et al., 1997). Moreover, the results of Brecht et al. (1997) raise the possibility that, in the texture discrimination tasks (Guic-Robles et al., 1989; Carvell and Simons, 1990), rats preferentially used the small rostralmost whiskers around the tip of the nose to solve the task and not the larger, caudal whiskers, a possibility that has never been tested. Although it is possible that the large mystacial vibrissae may subserve both functions (texture discrimination and distance detection), no previous study has systematically demonstrated that rats can discriminate small differences in distance using only these whiskers.

The results of the present study clearly demonstrate that the vibrissal array can function as a fine-grained distance detector. Rats rapidly learn to discriminate between apertures of very similar width (62 vs $65 \mathrm{~mm}$; a difference of $~ 5 \%$ ). Interestingly, Carvell and Simons (1995) reported that a $6 \%$ difference in the spacing of a fine tactile grating ( $1.00 \mathrm{vs} 1.06 \mathrm{~mm})$ appeared to be near the limits of tactile resolution in a very different whiskerdependent discrimination task. In the present study, rats performed aperture discriminations using only their large mystacial vibrissae. There was no evidence that they used the smaller whiskers around their nose or lips to perform the task. Moreover, it is very likely that rats could perform even finer discriminations if two different apertures were simultaneously presented side-byside in a two-choice discrimination paradigm instead of the single-aperture presentation used in the present study.

Furthermore, rats were also capable of discriminating aperture width quite rapidly. Typically, the whiskers of the rats remained in contact with the aperture for only a few hundred milliseconds, even during the most difficult discriminations. The time that whiskers remained in contact with the aperture was even shorter if rats were not also required to poke into the center nose poke to receive a reward. Also, rats rarely sampled the aperture more than once per trial, and they rarely lingered with their nose inside the aperture. Together, the speed with which rats are capable of performing these discriminations combined with their ability to discriminate very fine differences in aperture widths indicates that fine-grained distance detection is a normal function of the vibrissal array. Of course, as stated above, this does not preclude the possibility that rats might also use their large facial whiskers to discriminate between different surface textures.

\section{Multiwhisker integration}

The ability to perform discriminations of moderate difficulty ( 60 vs $68 \mathrm{~mm}$ ) requires $\sim 8-12$ intact whiskers on each side of the face. Moreover, systematic removal of individual whiskers resulted in a gradual decrease in the accuracy of the discrimination that was proportional to the number of whiskers removed. These results indicate that tactual information from many whiskers must be integrated to discriminate between different aperture widths accurately. This is consistent with (and further extends) the 
results of a previous report indicating that rats require at least two or more whiskers (per side) to discriminate between two very similar fine tactile gratings (Carvell and Simons, 1995). These results, which indicate that integration of tactual input from many facial whiskers is necessary for identifying complex tactile stimuli, are consistent with electrophysiological recording studies that demonstrate that individual neurons in the trigeminal somatosensory system receive tactile input from many facial whiskers (above).

Furthermore, the results of removing whiskers in different patterns (ROWS INTACT vs ARCS INTACT) indicate that tactile input derived from individual whiskers was functionally equivalent. Although rats in each of these groups had very different complements of whiskers at different stages of this experiment, the ability to discriminate did not differ. Also, the fact that whiskers were removed in patterns that preferentially left whisker rows or arcs intact indicates that these particular substructures of the vibrissal array are not functionally distinct, at least within the context of our behavioral paradigm. Furthermore, the results of the cortical lesion studies indicate that the ability to discriminate accurately is dependent on the SI cortex. Collectively, these results indicate that, as rats sample complex tactile stimuli, tactual signals from many, functionally similar whiskers are integrated by the trigeminal system as internal representations of the stimuli are constructed.

\section{Role of whisker movement during discrimination}

When rats explore novel objects or environments, they engage in a cluster of movements that include (1) polypnea, (2) protraction and retraction of the mystacial vibrissae ("whisking"), (3) head movements and fixations, and (4) protraction and retraction of the tip of the nose (Welker, 1964). Furthermore, rats trained to discriminate between different surface textures (Guic-Robles et al., 1989; Carvell and Simons, 1990) or to locate small objects in an open field (Brecht et al., 1997) with their mystacial vibrissae use this characteristic whisking motion of the facial whiskers as they palpate the surface. Together, these results indicate that whisking is used by rats to gather tactual information about their environment. However, no previous study has systematically examined whether whisking is necessary for rats to obtain finegrained tactile information with their large mystacial vibrissae.

The results of the present study demonstrate that rats are capable of performing very fine-grained tactile discriminations without active whisking motions. Video analysis of rats performing the task showed no evidence that rats used whisking to sample the aperture, even during the earliest training sessions when rats were initially learning the discrimination or during discriminations of very similar aperture widths. This was confirmed by the finding that cutting the facial nerve did not impair performance. Moreover, rats were capable of performing these fine-grained discriminations very rapidly, suggesting that acquisition of behaviorally useful tactual information without whisking is an intrinsic capability of the vibrissal system.

It should be noted that, although rats did not actively whisk the aperture, they did move rapidly into the aperture as it was sampled, resulting in a relative movement of the whiskers across the aperture. Thus, it is likely that some movement of the whiskers over the aperture is necessary for accurate discrimination. However, these results demonstrate that rats are capable of deriving fine-grained tactual information from the vibrissal array without active whisking and suggest that this may be a relatively common mode of function for the vibrissal array.
The finding that rats can accurately gauge aperture width without active whisking is, perhaps, not surprising. Video analysis of rats discriminating a textured surface by whisking the surface indicates that there is a relatively large amount of variation in which individual whiskers contact the surface on each successive sweep of the whiskers (Carvell and Simons, 1990, 1995). This variability in whisker motions and contacts may not be detrimental for texture discrimination; perhaps it is even beneficial. However, such variability would likely be detrimental for rats discriminating between apertures of similar width. Because the relative length of individual whiskers within the vibrissal array varies in a highly conserved manner (Brecht et al., 1997), minimizing variations in which these whiskers contact the apertures would maximize the potential to exploit this important anatomical feature of the vibrissal array. Presumably, in the aperture discrimination, maximal information could be obtained by minimizing variations in whisker position from trial to trial. The present finding that rats accurately perform the discrimination without any significant active movements supports this possibility. Collectively, these results suggest that rats may use different behavioral strategies as they sample different tactile stimuli, active whisking for finegrained texture discriminations and more fixed positioning of the vibrissae during fine-grained distance detection.

\section{Summary}

Here, we describe a novel behavioral task that was designed to facilitate quantitative analysis of the functional properties of the trigeminal somatosensory system in the awake behaving rat. Results of the experiments described here indicate that the vibrissal array intrinsically functions as a fine-grained distance detector without the need for active whisking. Accurate distance detection requires integration of complex tactual input from many, functionally equivalent whiskers, and the barrel cortex is critically involved in this discrimination.

\section{REFERENCES}

Armstrong-James M, Fox K (1987) Spatiotemporal convergence and divergence in the rat S1 "barrel" cortex. J Comp Neurol 263:265-281.

Arvidsson J (1982) Somatotopic organization of vibrissae afferents in the trigeminal sensory nuclei of the rat studied by transganglionic transport of HRP. J Comp Neurol 211:84-92.

Belford GR, Killackey HP (1979) Vibrissae representation in subcortical trigeminal centers of the neonatal rat. J Comp Neurol 183:305-321.

Brecht M, Preilowski B, Merzenich MM (1997) Functional architecture of the mystacial vibrissae. Behav Brain Res 84:81-97.

Carvell GE, Simons DJ (1990) Biometric analyses of vibrissal tactile discrimination in the rat. J Neurosci 10:2638-2648.

Carvell GE, Simons DJ (1995) Task- and subject-related differences in sensorimotor behavior during active touch. Somatosens Mot Res 12:1-9.

Chapin JK (1986) Laminar differences in sizes, shapes, and response profiles of cutaneous receptive fields in the rat SI cortex. Exp Brain Res 62:549-559.

Chiaia NL, Rhoades RW, Fish SE, Killackey HP (1991) Thalamic processing of vibrissal information in the rat. II. Morphological and functional properties of medial ventral posterior nucleus and posterior nucleus neurons. J Comp Neurol 314:217-236.

Dörfl J (1985) The innervation of the mystacial region of the white mouse. A topographical study. J Anat 142:173-184.

Ghazanfar AA, Nicolelis MA (1999) Spatiotemporal properties of layer $\mathrm{V}$ neurons of the rat primary somatosensory cortex. Cereb Cortex 9:348-361.

Ghazanfar AA, Nicolelis MA (2001) The structure and function of dynamic cortical and thalamic receptive fields. Cereb Cortex 11:183-193.

Guic-Robles E, Valdivieso C, Guajardo G (1989) Rats can learn a roughness discrimination using only their vibrissal system. Behav Brain Res 31:285-289.

Guic-Robles E, Jenkins WM, Bravo H (1992) Vibrissal roughness discrimination is barrel cortex-dependent. Behav Brain Res 48:145-152.

Gustafson JW, Felbain-Keramidas SL (1977) Behavioral and neural ap- 
proaches to the function of the mystacial vibrissae. Psychol Bull 84:477-488.

Hurwitz BE, Dietrich WD, McCabe PM, Watson BD, Ginsberg MD, Schneiderman N (1990) Sensory-motor deficit and recovery from thrombotic infarction of the vibrissal barrel-field cortex. Brain Res 512:210-220

Hutson KA, Masterton RB (1986) The sensory contribution of a single vibrissa's cortical barrel. J Neurophysiol 56:1196-1223.

Ito M (1981) Some quantitative aspects of vibrissa-driven neuronal responses in rat neocortex. J Neurophysiol 46:705-715.

Ito M (1985) Processing of vibrissa sensory information within the rat neocortex. J Neurophysiol 54:479-490.

Killackey HP (1973) Anatomical evidence for cortical subdivisions based on vertically discrete thalamic projections from the ventral posterior nucleus to cortical barrels in the rat. Brain Res 51:326-331.

Krupa DJ, Ghazanfar AA, Nicolelis MA (1999) Immediate thalamic sensory plasticity depends on corticothalamic feedback. Proc Natl Acad Sci USA 96:8200-8205.

Land PW, Simons DJ (1985) Metabolic and structural correlates of the vibrissae representation in the thalamus of the adult rat. Neurosci Lett 60:319-324.

Moore CI, Nelson SB (1998) Spatio-temporal subthreshold receptive fields in the vibrissa representation of rat primary somatosensory cortex. J Neurophysiol 80:2882-2892.

Moore CI, Nelson SB, Sur M (1999) Dynamics of neuronal processing in rat somatosensory cortex. Trends Neurosci 22:513-520.

Nicolelis MA, Chapin JK (1994) Spatiotemporal structure of somatosensory responses of many-neuron ensembles in the rat ventral posterior medial nucleus of the thalamus. J Neurosci 14:3511-3532.

Paxinos G, Watson C (1986) The rat brain in stereotaxic coordinates. San Diego: Academic.

Peterson BE, Goldreich D, Merzenich MM (1998) Optical imaging and electrophysiology of rat barrel cortex. I. Responses to small singlevibrissa deflections. Cereb Cortex 8:173-183.
Pinto DJ, Brumberg JC, Simons DJ (2000) Circuit dynamics and coding strategies in rodent somatosensory cortex. J Neurophysiol 83:1158-1166

Shuler MG, Krupa DJ, Nicolelis MAL (2000) Discrimination of bilateral whisker stimuli in the freely behaving rat. Soc Neurosci Abstr 20:366.318.

Simons DJ (1978) Response properties of vibrissa units in rat SI somatosensory neocortex. J Neurophysiol 41:798-820.

Simons DJ (1985) Temporal and spatial integration in the rat SI vibrissa cortex. J Neurophysiol 54:615-635.

Simons DJ (1995) Neuronal integration in the somatosensory whisker/ barrel cortex. In: Cereb cortex, Vol 11 (Jones EG, Diamond IT, eds), pp 263-297. New York: Plenum.

Vincent SB (1912) The function of the vibrissae in the behavior of the white rat. Behav Monographs 1:1-82.

Waite PM (1973) The responses of cells in the rat thalamus to mechanical movements of the whiskers. J Physiol (Lond) 228:541-561.

Waite PME, Tracey DJ (1995) Trigeminal sensory system. In: The rat nervous system (Paxinos G, ed), pp 705-724. San Diego: Academic

Welker C (1971) Microelectrode delineation of fine grain somatotopic organization of $(\mathrm{SmI})$ cerebral neocortex in albino rat. Brain Res 26:259-275.

Welker WI (1964) Analysis of sniffing of the albino rat. Behaviour 22:223-244.

Woolsey TA, Van der Loos H (1970) The structural organization of layer IV in the somatosensory region (SI) of mouse cerebral cortex. The description of a cortical field composed of discrete cytoarchitectonic units. Brain Res 17:205-242.

Zhu JJ, Connors BW (1999) Intrinsic firing patterns and whisker-evoked synaptic responses of neurons in the rat barrel cortex. J Neurophysiol 81:1171-1183.

Zucker E, Welker WI (1969) Coding of somatic sensory input by vibrissae neurons in the rat's trigeminal ganglion. Brain Res 12:138-156. 